\title{
Perfluorocarbon nanodroplets can reoxygenate hypoxic tumors in vivo without carbogen breathing
}

\author{
Yun Xiang $1^{*}$, Nicholas Bernards $2^{*}$, Bryan Hoang1,2, Jinzi Zheng2,3 and Naomi Matsuura1,3, ${ }^{\bowtie}$ \\ 1. Department of Medical Imaging, University of Toronto, Ontario, Canada \\ 2. TECHNA Institute for the Advancement of Technology for Health, University Health Network, Toronto, Ontario, Canada \\ 3. Institute of Biomaterials and Biomedical Engineering, University of Toronto, Ontario, Canada \\ 4. Department of Materials Science and Engineering, University of Toronto, Ontario, Canada \\ *These authors contributed equally to this work. \\ $\triangle$ Corresponding author: Naomi Matsuura, T: (416) 978-3681, E: naomi.matsuura@utoronto.ca \\ (c) Ivyspring International Publisher. This is an open access article distributed under the terms of the Creative Commons Attribution (CC BY-NC) license \\ (https://creativecommons.org/licenses/by-nc/4.0/). See http://ivyspring.com/terms for full terms and conditions.
}

Received: 2018.09.13; Accepted: 2019.02.08; Published: 2019.03.11

\begin{abstract}
Nanoscale perfluorocarbon (PFC) droplets have enormous potential as clinical theranostic agents. They are biocompatible and are currently used in vivo as contrast agents for a variety of medical imaging modalities, including ultrasound, computed tomography, photoacoustic and ${ }^{19} \mathrm{~F}$-magnetic resonance imaging. PFC nanodroplets can also carry molecular and nanoparticulate drugs and be activated in situ by ultrasound or light for targeted therapy. Recently, there has been renewed interest in using PFC nanodroplets for hypoxic tumor reoxygenation towards radiosensitization based on the high oxygen solubility of PFCs. Previous studies showed that tumor oxygenation using PFC agents only occurs in combination with enhanced oxygen breathing. However, recent studies suggest that PFC agents that accumulate in solid tumors can contribute to radiosensitization, presumably due to tumor reoxygenation without enhanced oxygen breathing. In this study, we quantify the impact of oxygenation due to PFC nanodroplet accumulation in tumors alone in comparison with other reoxygenation methodologies, in particular, carbogen breathing.

Methods: Lipid-stabilized, PFC (i.e., perfluorooctyl bromide, $\mathrm{CF}_{3}\left(\mathrm{CF}_{2}\right)_{7} \mathrm{Br}$, $\mathrm{PFOB}$ ) nanoscale droplets were synthesized and evaluated in xenograft prostate (DU145) tumors in male mice. Biodistribution assessment of the nanodroplets was achieved using a fluorescent lipophilic indocarbocyanine dye label (i.e., Dil dye) on the lipid shell in combination with fluorescence imaging in mice ( $n \geq 3$ per group). Hypoxia reduction in tumors was measured using PET imaging and a known hypoxia radiotracer, [18F]FAZA $(n \geq 3$ per group).

Results: Lipid-stabilized nanoscale PFOB emulsions (mean diameter of $\sim 250 \mathrm{~nm}$ ), accumulated in the xenograft prostate tumors in mice 24 hours post-injection. In vivo PET imaging with [18F]FAZA showed that the accumulation of the PFOB nanodroplets in the tumor tissues alone significantly reduced tumor hypoxia, without enhanced oxygen (i.e., carbogen) breathing. This reoxygenation effect was found to be comparable with carbogen breathing alone.

Conclusion: Accumulation of nanoscale PFOB agents in solid tumors alone successfully reoxygenated hypoxic tumors to levels comparable with carbogen breathing alone, an established tumor oxygenation method. This study confirms that PFC agents can be used to reoxygenate hypoxic tumors in addition to their current applications as multifunctional theranostic agents.
\end{abstract}

Key words: perfluorocarbon, reoxygenation, tumor hypoxia, nanodroplets, PET imaging

\section{Introduction}

Perfluorocarbons (PFCs) are hydrocarbons with all or most of the hydrogen atoms replaced by fluorine atoms. PFCs are chemically and biologically inert, are both lipophobic and hydrophobic, and have high gas solubility. PFCs can be stabilized by lipids $[1,2]$, polymers [3-5], proteins [6,7], or fluorosurfac- 
tants $[8,9]$ to form nanoscale droplets $(<500 \mathrm{~nm}$ in diameter) for intravenous injection. These PFC droplets have been widely exploited as a theranostic tool in biomedicine. For example, various PFC nanodroplets have been used to label cells for tracking by ${ }^{19} \mathrm{~F}-\mathrm{MRI}[10,11]$, and droplets with metastable PFC cores have been formulated as droplet-to-bubble phase-shift agents for photoacoustic and ultrasound imaging [8,12-14] or for image-guided drug delivery $[15,16]$. Heteroatoms (e.g., bromide) present in PFC molecules offers X-ray attenuation for contrastenhanced CT imaging $[17,18]$. For therapy, dispersed PFC nanodroplets have been used in combination with MRI-guided focused ultrasound and laser light for targeted drug delivery $[4,5,19,20]$, vascular disruption [21,22] and tumor ablation [10,23-26].

One of the earliest applications of stabilized nanoscale PFC emulsions was to carry oxygen as red blood cell substitute agents [27,28]. These PFC emulsions were also used in other medical treatments, including the reoxygenation of hypoxic tumors. It is known that hypoxia is one of the major causes of radioresistance in many types of solid tumors [29-31], and tumor oxygenation has been positively correlated with radiotherapy efficacy [32-34]. Fluosol DA, an albumin-stabilized perfluorodecalin and perfluorotributylamine emulsion, was used in combination with enhanced oxygen (i.e., hyperbaric oxygen (HBO)/ carbogen) breathing to sensitize tumors to radiotherapy in preclinical models $[7,35,36]$ and in Phase I/II clinical trials [37-39]. Later, second-generation PFC agents were developed towards improved stability and ease of use. For example, Oxygent, a lipidstabilized perfluorooctyl bromide $\left(\mathrm{CF}_{3}\left(\mathrm{CF}_{2}\right)_{7} \mathrm{Br}\right.$, PFOB) nanoemulsion, similarly demonstrated tumor radiosensitization in preclinical tumor models $[2,40,41]$ that was presumed to be due to increased oxygenation in the tumor $[42,43]$. These results were attributed to the increased oxygen-carrying capacity of the PFCs in the bloodstream when applied with enhanced oxygen breathing (i.e., carbogen or $\mathrm{HBO}$ ). For optimal radiosensitization, the PFC agents and carbogen/HBO breathing needed to be administrated concurrently and immediately before radiotherapy delivery. It was found in both preclinical and clinical studies that if the PFC agents were used alone, limited radiosensitization was observed $[35,44]$.

More recently, led by multidisciplinary advances in cancer nanomedicine, several nanosized PFC emulsions have been designed to accumulate in solid tumors through the enhanced permeability and retention (EPR) effect [4,20,45-47]. When applied to hypoxic tumors, several recent studies have shown improved radiotherapeutic outcomes using PFC agents, even without $\mathrm{HBO} /$ carbogen breathing
[48-50]. The conventional strategy for PFC emulsion use relied on increasing the PFC concentration in systemic blood circulation as oxygen carriers and delivering radiotherapy soon after the injection of PFC emulsions. In contrast, in these recent studies, radiotherapy was delivered 24 hours after the administration of the PFC agents, thereby allowing sufficient time for PFC nanodroplets extravasation into tumors via the EPR effect. This new approach is exciting because this means that the same PFC agent validated for tumor imaging and therapy may also be used for radiosensitization. The use of injectable PFC agents for radiosensitization can reduce the toxicity and discomfort of alternative clinical tumor reoxygenation approaches (e.g., carbogen breathing) for patients. Also, the use of the EPR effect could substantially lower PFC agent doses for tumor radiosensitization to prevent acute hemodilution arising from the large PFC agent doses required to increase oxygen transportation in studies using Fluosol DA and Oxygent [51,52]. Finally, if reoxygenation using PFC nanoscale agents alone can be accomplished, PFC nanoscale agents can be combined with other methods that are currently used for reoxygenation and radiosensitization for greater therapeutic impact.

An unresolved question is whether the observed radiosensitization effect due to PFC nanosized agent administration without carbogen in previous studies was a result of tumor reoxygenation or some other mechanism. These previous preclinical studies did not measure tumor oxygen levels at all [49], or measured tumor hypoxia indirectly through non-quantitative immunohistochemical staining of hypoxia markers (e.g., hypoxia-inducible factor 1-alpha, carbonic anhydrase IX, and/or pimonidazole) post-sacrifice $[48,53]$, or measured hypoxia via the use of invasive Eppendorf microelectrodes that cannot accurately measure whole tumor oxygenation [44,49,54]. Moreover, it is known that PFC agents can sensitize tumors to radiotherapy by means other than direct tumor reoxygenation. For example, metastable PFC droplets are known to spontaneously vaporize at physiological temperature after their accumulation [55] and cavitation-induced vascular damage and cell death signaling initiated from damaged endothelium can facilitate tumor growth delay in combination with radiotherapy [56,57]. Also, even if PFC emulsions alone can directly oxygenate tumors, it is unclear how this approach compares with previously wellestablished oxygenation approaches (i.e., carbogen breathing alone).

In this study, we aim to determine if PFC nanoemulsions can oxygenate hypoxic tumors in the presence and absence of carbogen breathing in a 
xenograft mouse model of hypoxic human prostate cancer [58]. To directly and non-invasively quantify tumor oxygenation due to PFC nanoemulsions in vivo, we use positron emission tomography (PET) in combination with $\left[{ }^{18} \mathrm{~F}\right]$ fluoroazomycin-arabinoside ([18F]FAZA) as the hypoxia-specific tracer $[53,59]$. High boiling point and high stability lipid-stabilized PFOB nanoemulsions that are resistant to spontaneous in vivo cavitation and with known oxygenation capabilities were selected as the representative PFC agents to be evaluated [27].

\section{Method}

\section{Preparation and characterization of PFOB nanoemulsions}

PFC nanoemulsions were prepared from PFOB (SynQuest Laboratories, Alachua, FL, USA), soybean lecithin (Fisher Scientific, Ottawa, ON, Canada) and 1,2-dipalmitoyl-sn-glycero-3-phosphoethanolamine$\mathrm{N}$-[methoxy (polyethylene glycol)- 2000] (ammonium salt) (DPPE-PEG2000, Avanti Lipids, Alabaster, AL, USA). Briefly, lecithin and DPPE-PEG2000 were mixed and dissolved in chloroform (VWR, Radnor, PA, USA) at a mass ratio of 5:13 (DPPE-PEG2000: lecithin) to a total lipid concentration of $18 \mathrm{mg} / \mathrm{mL}$. This lipid solution was evaporated in a round-bottom flask to form a dry lipid-film using rotary evaporation (R215, Buchi, New Castle, DE, USA) at 100 mBar. Fluorescently labeled PFOB nanoemulsions were prepared using fluorescent lipophilic indocarbocyanine dye, DiI (Sigma-Aldrich, Oakville, ON, Canada), pre-dissolved in diethyl ether and mixed with the lipid solution in chloroform before evaporation to load the hydrophobic molecules on the shell of the PFOB nanodroplets [60]. The dried lipid film was rehydrated using deionized water and sonicated in a bath sonicator (2510 ultrasonic cleaner, Branson Ultrasonics, Danbury, CT, USA) for 10 minutes to disperse the lipids in the water phase. PFOB was added to the lipid suspension with a mass ratio of 4:1 (water: PFOB) and this two-phase mixture was emulsified using a probe Sonifier with a $1 / 8^{\prime \prime}$ microtip (S-250 Digital Sonifier, Branson, USA) at $10 \%$ power output (with 1-sec.-on-1-sec.-off pulse) in an ice water bath for a total sonication time of $10 \mathrm{~min}$. The PFOB nanoemulsion was then centrifuged at 4000 RPM for 10 min (Centrifuge 5430, Eppendorf, Hamburg, Germany). After centrifugation, the supernatant was then removed and the PFOB nanodroplet pellet was resuspended in deionized water. This wash process was repeated twice more to remove free lipids, micelles, liposomes and small droplets in the supernatant. The final emulsion was reconstituted in $0.9 \%$ saline and filtered through a 0.45 um syringe filter (Pall Corporation, Port Washington, New York, USA) to remove larger droplets or aggregates. The prepared $\mathrm{PFOB}$ nanoemulsion was stored at $4^{\circ} \mathrm{C}$ until use.

Size-distribution, mean diameter and the polydispersity index (PDI) of both the plain and DiI-labeled PFOB nanoemulsions were acquired at room temperature using a dynamic light scattering (DLS) particle sizer (Malvern Zetasizer Nano-ZS 3000HS, Worcestershire, U.K.). The fluorescence emission of DiI was measured using a Horiba Jobin Yvon FluoroMax-4 Spectrofluorometer (Horiba Scientific, Edison, NJ, USA) at an excitation wavelength of $549 \mathrm{~nm}$. For stability assessment, both plain and DiI-labeled PFOB nanoemulsions were stored as-formulated (200 mg PFOB per $\mathrm{mL}$ ) and in diluted form ( $1 \mathrm{mg}$ PFOB per $\mathrm{mL}$ ) at different temperatures $\left(4^{\circ} \mathrm{C}, 22{ }^{\circ} \mathrm{C}\right.$, and $\left.37^{\circ} \mathrm{C}\right)$ up to 20 days.

\section{Animal model for in vivo assessment}

An androgen-independent human prostate tumor cell line (DU145) was obtained from ATCC (Manassas, VA, USA). Cells were cultured in Dulbecco's modified Eagle medium with $10 \%$ (v/v) fetal bovine serum, $100 \mathrm{U} / \mathrm{ml}$ penicillin and 0.1 $\mathrm{mg} / \mathrm{mL}$ streptomycin (Gibco, ThermoFisher, Waltham, Massachusetts, USA). The cells were grown in a humidified $5 \% \mathrm{CO}_{2}$ atmosphere at $37^{\circ} \mathrm{C}$. Cells were detached from the flasks for subculture using $0.25 \%$ trypsin and $0.02 \%$ EDTA (ThermoFisher) when the cell growth reached $80 \%$ confluency.

Radioresistant NOD-SCID mice (NRG) purchased from the Ontario Cancer Institute (Toronto, $\mathrm{ON}$ ) were inoculated with $5 \times 10^{6}$ cells in a volume of $50 \mu \mathrm{L}$ subcutaneously into the left flank. NRG mice were selected for compatibility with later radiosensitization studies. Animals were housed under regulated temperature and humidity $(40-60 \%)$ conditions and light/dark cycles (12h on and $12 \mathrm{~h}$ off) with free access to food and water. Caliper measurements of tumor length (L) and width (W) were performed every three days. The tumor volume $(\mathrm{V})$ was obtained using the formula $V=\left(\mathrm{W}^{2} \times \mathrm{L}\right) / 2$ [61].

\section{Fluorescence imaging for biodistribution assessment}

The mice were randomized into three groups for biodistribution assessment after the average tumor volume reached $210 \mathrm{~mm}^{3}$. All mice were anesthetized under isoflurane through face masks. In all experiment groups, $170 \mu \mathrm{L}$ DiI-labeled PFOB nanoemulsions $(100 \mathrm{w} / \mathrm{v} \%$ of PFOB to a final dose of $10 \mathrm{~g}$ PFOB $/ \mathrm{kg}$ ) was delivered via i.v. injection. Groups I $(n=3)$, II $(n=4)$, and III $(n=3)$ were sacrificed at $3 \mathrm{~h}, 6$ $\mathrm{h}$, and $24 \mathrm{~h}$ post PFOB nanoemulsion injection, 
respectively. The heart, lung, spleen, left kidney, liver, muscle, and tumor were resected from each animal and immediately imaged using a Maestro hyperspectral fluorescence system (Cambridge Research \& Instrumentation, Inc (Cri), Woburn, MA, USA) selected to measure DiI's secondary emissions peak at $610 \mathrm{~nm}$ (Supplementary Figure S1). All exposures were determined automatically to avoid any saturation. The emissions filter range was set from $550-660 \mathrm{~nm}$ and all samples were placed central to the field-of-view, and image acquisition occurred immediately after organ resection. The regions of interest (ROIs) were drawn over each organ and analyzed using 2D fluorescence imager (Maestro, Cri Version 2.10.0) [62-65].

\section{In vivo PET imaging of hypoxia assessment}

In this study, FAZA-PET image acquisitions took place once the tumors reached an average volume of $254 \mathrm{~mm}^{3}$. The animals were randomized into four groups: Group $1(\mathrm{n}=3)$ was the untreated control group, group $2(n=4)$ received carbogen for $2 h$ prior to imaging, group $3(n=3)$ was injected with $P F O B$ nanoemulsion $24 \mathrm{~h}$ before imaging, and group $4(\mathrm{n}=4)$ were injected with PFOB nanoemulsion $24 \mathrm{~h}$ before imaging and received carbogen for $2 \mathrm{~h}$ prior to imaging. The experimental groups 3 and 4 were administrated $170 \mu \mathrm{L}$ of PFOB nanoemulsions $(10 \mathrm{~g}$ $\mathrm{PFOB} / \mathrm{kg}$ ) via i.v. tail-vein injection $24 \mathrm{~h}$ prior to imaging as this was the time point for maximum PFOB nanoemulsion accumulation as determined in the biodistribution study.

The PET hypoxia tracer, $\left[{ }^{18} \mathrm{~F}\right] \mathrm{FAZA}$, used in this study was produced by CanProbe (Toronto, ON, Canada) using a TRACERlab FXFN (GE Healthcare) automated synthesis unit with good manufacturing practices and Health Canada standards and had an overall average yield of $10 \%$ at the end of synthesis. The radiochemical purity was always $>95 \%$ at the release of the product and all other quality control specifications were met $[66,67]$. The mice were injected with $\sim 13 \mathrm{MBq}\left[{ }^{18} \mathrm{~F}\right] \mathrm{FAZA}$ in a volume of $\sim 100$ $\mu \mathrm{L}$. PET acquisitions were obtained using a FOCUS $220 \mu$ PET (Siemens, USA). Acquisitions started at $2 \mathrm{~h}$ post-injection of $\left[{ }^{18} \mathrm{~F}\right] \mathrm{FAZA}$ as this has been deemed the ideal time point at which $\left[{ }^{18} \mathrm{~F}\right] \mathrm{FAZA}$ accumulation reaches steady-state within the tumor area [53]. The acquisition lasted 20 minutes for the emission scan [68] (Supplementary Figure S2). Attenuation correction was carried out using an 8-minute transmission scan over an external radioactive positron-emitting source $\left({ }^{57} \mathrm{Cu}\right.$ source). CT images were obtained using a GE Locus Ultra $(80 \mathrm{kVp}$ and 50 $\mathrm{mA}$ ) immediately post PET acquisition using the same animal bed, to facilitate accurate co-registration between the anatomical (CT) and molecular (PET) modalities. Images were later co-registered and analyzed using IRW (Inveon Reseach Workplace, version 4.0, Siemens). ROIs were determined using CT data to accurately delineate and quantify tumor margins.

\section{Results and Discussion}

\section{PFOB nanoemulsion synthesis and characterization}

PFOB was selected for this study because of its previous use in patients as oxygen carriers [27] and its strong potential in other tumor imaging and therapy applications [69-72]. Also, compared to pure PFC materials used in other studies, the higher boiling point and the increased lipophilicity of PFOB hinders coalescence and spontaneous vaporization such that stable nanoscale droplets can be evaluated in vivo [27].

The PFOB nanodroplets consisted of a PFOB core surrounded by a lipid shell comprised of a mixture of lecithin and DPPE-PEG (Figure 1A). The average hydrodynamic mean diameter (i.e., Z-average) of the PFOB nanodroplets as formulated were $208 \pm 10 \mathrm{~nm}$ with a PDI $=0.19 \pm 0.01 \quad(n=6)$. After centrifugation to remove smaller droplets, liposomes and micelles formed by excess lipids, the mean diameter increased to $254 \pm 7 \mathrm{~nm}$, with improved monodispersity (PDI= $0.11 \pm 0.01)(\mathrm{n}=6)$. After filtration to remove larger droplets, the mean diameter remained unchanged but the PDI was further reduced to below 0.1 (Figure 1B, Table 1), indicating a narrowing size distribution. Incorporation of the lipophilic dye (i.e., to a final concentration of $0.5 \mathrm{mg} / \mathrm{mL}$ Dil in PFOB) did not significantly change the mean size or PDIs (Figure 1B, Table 1). The final mean size after purification determined by DLS is comparable with other PFOB emulsions prepared using similar procedures $[3,71,73,74]$.

Table 1. The mean size (Z-average) and PDI of both plain and Dil-labeled nanodroplets through the preparation and purification procedure.

\begin{tabular}{lllll}
\hline Sample & & $\begin{array}{l}\text { Post- } \\
\text { sonication }\end{array}$ & $\begin{array}{l}\text { Post- } \\
\text { centrifugation }\end{array}$ & $\begin{array}{l}\text { Post- } \\
\text { filtration }\end{array}$ \\
\hline Plain & Diameter $(\mathrm{nm})$ & $207 \pm 9$ & $254 \pm 7$ & $254 \pm 4$ \\
nanodroplets & PDI & $0.19 \pm 0.01$ & $0.11 \pm 0.01$ & $0.08 \pm 0.01$ \\
Labeled & Diameter $(\mathrm{nm})$ & $206 \pm 11$ & $255 \pm 7$ & $259 \pm 4$ \\
nanodroplets & PDI & $0.17 \pm 0.02$ & $0.10 \pm 0.02$ & $0.08 \pm 0.01$ \\
\hline
\end{tabular}

The size stability of both plain PFOB nanodroplets and DiI-loaded PFOB nanodroplets were analyzed over a 20-day period at $4{ }^{\circ} \mathrm{C}$ (storage temperature), $22{ }^{\circ} \mathrm{C}$ (room temperature), and $37^{\circ} \mathrm{C}$ (physiological temperature) ( $\mathrm{n}=3$ for each condition). The samples stored at $4{ }^{\circ} \mathrm{C}$ were more stable, with 
average sizes increasing from $250 \pm 5 \mathrm{~nm}$ to $316 \pm 13$ $\mathrm{nm}$ over the 20-day period, compared to the samples stored at $22{ }^{\circ} \mathrm{C}$ and $37{ }^{\circ} \mathrm{C}$, where the average sizes increased to $345 \pm 27 \mathrm{~nm}$ and $390 \pm 25 \mathrm{~nm}$, respectively. Diluted samples were found to be less stable than the concentrated samples at $22{ }^{\circ} \mathrm{C}$ and 37 ${ }^{\circ} \mathrm{C}$, with the mean sizes increasing to $409 \pm 23 \mathrm{~nm}$ and $434 \pm 26 \mathrm{~nm}$, respectively (Figure 1C). However, no substantial increase in size was observed between the groups at 24 hours (Figure 1C), the expected time frame for maximal PFOB accumulation in tumors $[49,50]$.

Similar to previously published data, although bromination of the fluorinated molecule can increase the inherent lipophilicity of the PFC, the mean size of the prepared emulsion still significantly increased after long-term storage [75-77]. It is possible to increase stability by adding perfluorodecyl bromide
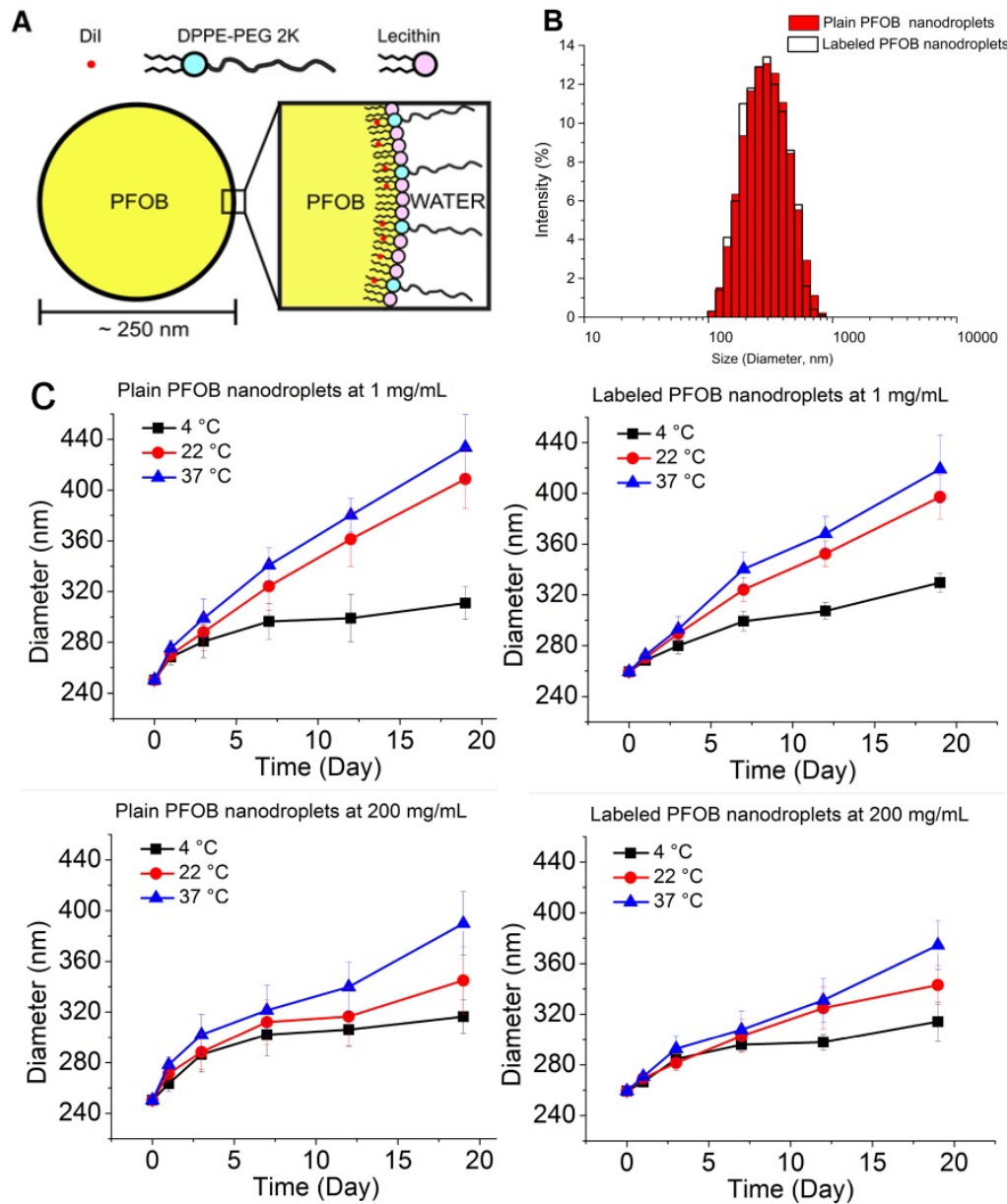

Figure 1. (A) Schematic of Dil-loaded, PFOB nanodroplet with a PFOB core and a lipid shell comprised of lecithin and DPPE-PEG2000. The hydrophobic tails of lipids face inward towards the slightly lipophilic PFOB, while the hydrophilic heads of the lipids face outward along with the water-soluble PEG chains. (B) Hydrodynamic mean diameter (Z-average) distribution of both plain and Dil-labeled PFOB nanodroplet as measured by DLS immediately after preparation and purification. (C) Size stability of both plain and Dil-labeled PFOB nanodroplet at two different concentrations, $1 \mathrm{mg} / \mathrm{mg}$ and $200 \mathrm{mg} / \mathrm{mL}$. All samples were tested at three temperatures $\left(22^{\circ} \mathrm{C}\right.$, $37^{\circ} \mathrm{C}$, and $4{ }^{\circ} \mathrm{C}$ ) for 20 days. Error bars indicate the standard deviation of the replicated samples $(n=3)$.
(PFDB), a fluorocarbon-hydrocarbon diblock compound, to the PFOB core to reduce Ostwald ripening, the primary cause of the emulsion destabilization during long-term storage [27]. To counteract the expected nanodroplet size changes over time, for all in vivo studies conducted here, the PFOB nanoemulsion were prepared the day of injection and all the animal experiments were limited to approximately 24 hours.

\section{PFOB nanodroplet biodistribution assessment}

Fluorescent imaging was applied to determine the biodistribution of DiI-labeled PFOB nanodroplets in vivo. The prostate tumor cell line DU145 has been widely used to establish hypoxic tumors [58]. The fluorescence of resected hearts, kidneys, lungs, livers, muscle, spleens, and tumors of mice bearing DU145 subcutaneous tumor xenografts $3 \mathrm{~h}, 6 \mathrm{~h}$, and $24 \mathrm{~h}$ postinjection of Dil-labeled PFOB nanodroplets were measured (Figure 2A). Three hours post-injection, uptake of the DiIlabelled PFOB nanodroplets were predominantly localized within the liver, spleen, and lungs; primary organs of the reticuloendothelial system responsible for clearance of nanoscale agents. Consistent with previous observations, nanoscale agents were preferentially taken up by the liver, spleen, and lungs in decreasing concentration, respectively [78]. In comparison, limited uptake of DiI-labelled PFOB nanodroplets in the heart, kidney and muscle tissue was observed. Maximal hepatic uptake occurred at $3 \mathrm{~h}$ and decreased by approximately half at 6 hours. In contrast, tumor uptake of the nanodroplets increased 3-fold from $6 \mathrm{~h}$ to $24 \mathrm{~h}$ post-injection with the maximum uptake occurring at $24 \mathrm{~h}$ (Figure 2B). This short-term biodistribution assessment was consistent with our previous studies of similar PFC materials [1]. Moreover, the $24 \mathrm{~h}$ time point confirmed a significant increase in PFOB nanodroplet accumulation in the tumor, which was consistent with recent radiosensitization studies using advanced PFC emulsion administration [48-50]. Thus, the $24 \mathrm{~h}$ time point was selected for subsequent in vivo tumor reoxygenation assessment.

\section{In vivo hypoxia tumor reoxygenation}

PET/CT imaging provided unique insight into the whole-body distribution of $\left.{ }^{18} \mathrm{~F}\right] \mathrm{FAZA} 24$ hours post-injection of 
PFOB nanoemulsions, with and without carbogen breathing and untreated controls. The tumor and reference tissues were contoured after co-registration of the PET images with CT to quantitatively measure the FAZA distribution (Figure 3A). Coronal, sagittal, and axial segmented images of a central tumor slice from a representative animal from each group is shown in Supplementary Figure S3. The FAZA signal was normalized as the percentage of injected dose (decay corrected) per gram tissue (\%ID/g) (Figure $3 B)$. In addition to the tumor, FAZA signals from muscle and the heart were also included as well-oxygenated controls. A significant reduction in tumor hypoxia for the group of animals which received PFOB nanoemulsions administered 24 hours prior to PET imaging was found compared to the non-treated controls $(\mathrm{p}<0.05)$. No significant change in hypoxia was found in the heart or muscle tissue across treatment groups. Of significance, the level of hypoxia reduction due to the PFOB nanoemulsions alone was similar to that of the group breathing carbogen alone $(p>0.90)$. This observed decrease in the $\left[{ }^{18} \mathrm{~F}\right] \mathrm{FAZA}$ signal demonstrates that the administration of PFOB nanoemulsions can significantly decrease hypoxia in solid tumors after their passive accumulation into tumors, even without carbogen breathing. In the presence of carbogen breathing, a further significant decrease in [ $\left.{ }^{18} \mathrm{~F}\right] \mathrm{FAZA}$ uptake in the tumors was observed $(\mathrm{P}<0.05)$ indicating that the combination of $\mathrm{PFOB}$ nanoemulsions and carbogen breathing can further ameliorate tumor hypoxia. However, as the hypoxia level in the tumor after carbon breathing alone and PFOB nanoemulsions alone were close to background levels as found in muscle and heart tissue, a significant decrease in tumor hypoxia was not found.

In addition to comparing the absolute signal from $\left[{ }^{18} \mathrm{~F}\right] \mathrm{FAZA}$, normalized data presented in the form of tumor-to-muscle (T/M) ratios for each animal is shown to rule out the influence of differences between individual animals (Figure 3C). The average $\mathrm{T} / \mathrm{M}$ ratio of the group receiving $\mathrm{PFOB}$ nanoemulsion alone and carbogen breathing alone were comparable, with no significant difference between these two groups $(p=0.30)$ (Figure. 3B). However, both treated groups were significantly lower compared with the untreated control group $(\mathrm{p}<0.05)$. Furthermore, the group that received both carbogen breathing and PFOB nanoemulsions also did not demonstrate a reduced $\mathrm{T} / \mathrm{M}$ ratio compared with the groups of only carbogen breathing or only PFOB injection. That is because either PFOB nanoemulsion alone or carbogen alone already reduces the $\mathrm{T} / \mathrm{M}$ ratio back to 1 .

Since oxygenation of the hypoxic tumors was maintained after the nanoemulsions were cleared from the bloodstream [79] and without additional any enhanced oxygen breathing, this suggests that the enhanced radiotherapy observed in recent studies may indeed be attributed to the increase in tumor oxygenation conferred by PFC nanoemulsion administration alone [49]. This observed oxygenation effect is somewhat surprising because the total volume of the nanosized PFC emulsion trapped inside the tumor is expected to be very limited [49], so the oxygen dissolved in PFC nanodroplets trapped in the tumor will be low. Also, given that any oxygen dissolved in the PFC nanoemulsion is expected to immediately balance with the surrounding tissue and not be retained inside the nanoemulsions for extended periods of time (i.e., 24 hours), such significant reoxygenation of tumors without carbogen breathing is unexpected.
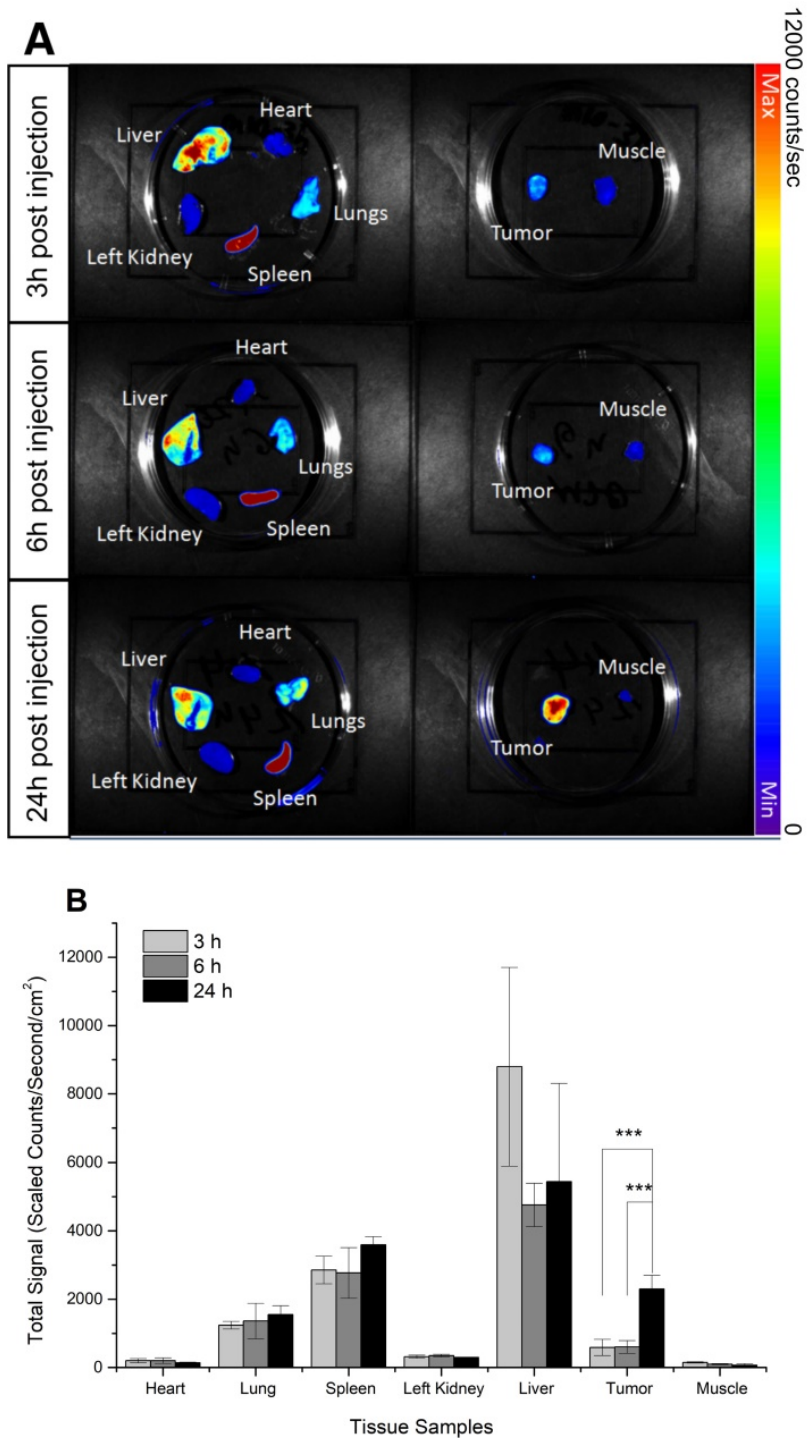

Figure 2. Biodistribution of Dil-labeled PFOB nanodroplets in NRG mice bearing DU145 tumor xenografts: (A) Representative ex vivo fluorescence images of organs and tumor tissues acquired at $3 \mathrm{~h}, 6 \mathrm{~h}$, and $24 \mathrm{~h}$ post-injection of PFOB nanodroplets.) (B) The quantitative fluorescence signal of Dil acquired from organs and tumors tissues acquired at $3 \mathrm{~h}, 6 \mathrm{~h}$, and $24 \mathrm{~h}$ post-injection $(\mathrm{n} \geq 3$, $\mathrm{p}$-value $<0.005$ from Tukey test was shown as $* * *)$. 

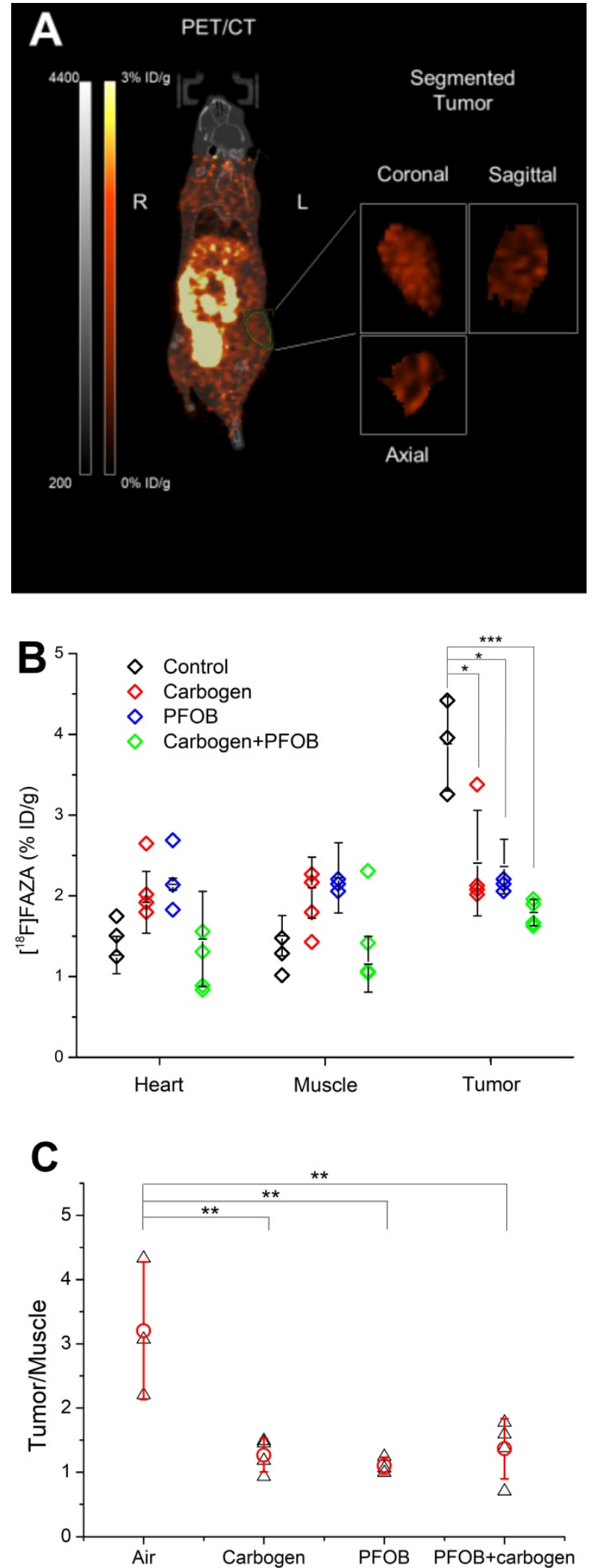

Figure 3. (A) For each animal, the $3 \mathrm{D}$ anatomical (CT) and molecular (PET) modalities were co-registered and the tissue segmentations including muscle, heart, and tumor were performed based on the anatomical image for quantitative hypoxia analysis. (B) The signal of [18F]FAZA (expressed in \%ID/g) from PET imaging detected in the muscle, heart, and tumor segmentation from each animal and the mean \pm standard deviation. (C) The normalized tumor-to-muscle ratio for each animal (Black triangles) and the mean ratio \pm standard deviation (Red circles) $(n \geq 3, p$ values $<0.05,<0.01$, and $<0.005$ from Tukey test was shown as *, **, and ***).
It is possible that the presence of PFC nanodroplets in the tumor alters the diffusion status of the oxygen in the tumor tissue. This is similar to what has been observed using PFCs in bioreactors or tissue engineering where mixing PFC nanoemulsions into a medium or a gel can significantly increase their oxygen diffusivity and solubility to keep encased cells alive [80,81]. Unlike hemoglobin, PFC droplets do not chemically chelate oxygen. Instead, PFCs dissolve oxygen with solubility directly proportional to the gas's partial pressure. Thus, the PFC droplets can release oxygen in the direction of lower oxygen partial tension (e.g., the hypoxic tumor) while it continually equilibrates with the relatively higher oxygen partial tension from the bloodstream, resulting in an increase in the tissue's oxygen permeability [82].

The Krogh model, a mathematical diffusion model which has been widely used to study and predict the oxygen diffusion pattern from a microvessel into surrounding tissues, can theoretically evaluate the oxygenation effect of PFC nanodroplets accumulation in a tumor [83-85]. However, according to this model, oxygenation of hypoxic tissue due to PFC nanodroplets can only become comparable with enhanced oxygen breathing when the amount of PFC material is both evenly distributed in the tissue and is greater than $8 \%$ of the total tumor volume (Supplementary Figure S4). However, since no more than $\sim 10 \%$ of total injected nanoscale agents typically accumulate inside solid tumors through the EPR effect [86], practically achieving such a high volume ratio in the tumor as predicted by the model is unlikely.

This discrepancy between the model prediction and experimental observations may be due to several reasons. First, although carbogen breathing definitively increases the oxygen partial tension in the blood circulation, it also causes other complicated systematic effects not captured by the simple model. Carbogen breathing changes the blood flow rate, heart rate, blood vessel diameter, oxygen affinity to the hemoglobin, the blood $\mathrm{pH}$, and the carbon dioxide transportation in the blood $[87,88]$, compromising the predicted oxygenation of the tumor. Second, in the model, the increased oxygen diffusivity is only based on the volume ratio of the PFC material in the tissue $[89,90]$. However, previous studies have shown that the size of the PFC droplets may play a crucial factor in oxygenation [73]. The smaller the PFC droplets in the emulsion, the larger its surface-to-volume ratio which can increase oxygen diffusion both into and out of the PFC nanodroplets. Furthermore, PFC droplet size will also impact biodistribution and accumulation in tumor tissue. For example, smaller droplets may have a greater chance to migrate deeper into the 
tumor rather than accumulate perivascularly, impacting oxygenation performance [45]. More detailed studies will be required to determine the relationship between tumor reoxygenation and PFC emulsion size.

PFOB agents are currently being extensively explored as tumor imaging or therapeutic agents. This study demonstrates the possibility to expand the use of this theranostic agent towards tumor reoxygenation as well, which can not only sensitize tumors to radiotherapy but also potentially to many other types of therapy including chemotherapy [31,91] and photodynamic therapy (PDT). For example, in type II PDT, a photosensitizer produces singlet oxygen $\left({ }^{1} \mathrm{O}_{2}\right)$ when irradiated by light of a specific wavelength to instantaneously damage biomolecules. Since this process also consumes oxygen in the tumor, aggravating hypoxia and limiting the therapeutic effect [92], there is great potential in directly replenishing the oxygen in the tumor using PFC nanodroplets. The quantitative measurement of oxygenation in a tumor due to PFC accumulation as determined in this study can guide the concurrent delivery of PFC nanodroplets with photosensitizers for PDT, through co-injection or even directly loading of photosensitizers onto PFC nanodroplets [93,94].

Furthermore, enhanced oxygen breathing is poorly tolerated by patients and is difficult to deliver consistently patient to patient [95]. Since PFOB nanoemulsions have already been tested as a blood substitute agent in patients [96], it may be possible to use this same agent in the clinic as a means to potentially overcome poor treatment efficacy of cancer therapy in hypoxic tumors alone or as a means to increase the efficacy of carbogen or $\mathrm{HBO}$ breathing [97].

\section{Conclusion}

This study demonstrated that lipid-stabilized PFOB nanodroplets approximately $250 \mathrm{~nm}$ in size can accumulate in the tumor 24 hours after administration and oxygenate hypoxic prostate cancer tumor xenografts in mice without carbogen breathing. Hypoxia was directly measured in vivo using $\left[{ }^{18} \mathrm{~F}\right] \mathrm{FAZA}-\mathrm{PET}$ imaging. This reoxygenation due to PFOB nanodroplet administration alone is comparable with carbogen breathing alone in the same model, which shows the potential of using this multimodal agent to replace carbogen breathing as a mean to sensitize hypoxic tumors to therapies including radiotherapy, chemotherapy, and PDT.

\section{Abbreviations}

[18F]FAZA: [ $\left.{ }^{18} \mathrm{~F}\right]$ fluoroazomycin-arabinoside; CT: Computed tomography; DLS: dynamic light scattering; DPPE-PEG2000: 1,2- dipalmitoyl- sn- glycero- 3- phosphoethanolamine- N- [methoxy (polyethylene glycol)-2000] (ammonium salt); EPR: enhanced permeability and retention; HBO: Hyperbaric oxygenation; PDI: polydispersity index; PET: positron emission tomography; PFC: Perfluorocarbon; PFDB: perfluorodecyl bromide; PFOB: perfluorooctyl bromide; MRI: Magnetic Resonance Imaging.

\section{Acknowledgments}

This project was supported by funds from Prostate Cancer Canada, the Movember Foundation (D2014-7), and the Ontario Research Fund, Early Researcher Award (ER14-10-178), the Canadian Cancer Society (703909), the Natural Sciences and Engineering Research Council of Canada (2015-058 35), the CFI-JELF program (36586) and the Strategic Training and Transdisciplinary Radiation Science for the 21st Century (STARS21) Program.

\section{Supplementary Material}

Supplementary figures and information. http://www.ntno.org/v03p0135s1.pdf

\section{Competing Interests}

The authors have declared that no competing interest exists.

\section{References}

1. Amir N, Green D, Kent J, et al. 18F-Labeled perfluorocarbon droplets for positron emission tomography imaging. Nucl Med Biol. 2017; 54: 27-33.

2. Thomas C, Riess J, Guichard M. Influence of the $100 \% \mathrm{w} / \mathrm{v}$ perfluorooctyl bromide (PFOB) emulsion dose on tumour radiosensitivity. Int J Radiat Biol. 1991; 59: 433-445.

3. Yao Y, Zhang M, Liu T, et al. Perfluorocarbon-Encapsulated PLGA-PEG Emulsions as Enhancement Agents for Highly Efficient Reoxygenation to Cell and Organism. ACS Appl Mater Interfaces. 2015; 7: 18369-18378.

4. Rapoport N, Nam K-H, Gupta R, et al. Ultrasound-mediated tumor imaging and nanotherapy using drug loaded, block copolymer stabilized perfluorocarbon nanoemulsions. J Control Release. 2011; 153: 4-15.

5. Rapoport N, Gao Z, Kennedy A. Multifunctional nanoparticles for combining ultrasonic tumor imaging and targeted chemotherapy. J Natl Cancer Inst. 2007; 99: 1095-106.

6. Song $\mathrm{X}$, Feng L, Liang $\mathrm{C}$, et al. Ultrasound Triggered Tumor Oxygenation with Oxygen-Shuttle Nanoperfluorocarbon to Overcome Hypoxia-Associated Resistance in Cancer Therapies. Nano Lett. 2016; 16: 6145-6153.

7. Chaplin DJ, Horsman MR, Aoki DS. Nicotinamide, Fluosol DA and Carbogen: a strategy to reoxygenate acutely and chronically hypoxic cells in vivo. Br J Cancer. 1991; 63: 109-13.

8. Williams R, Wright C, Cherin E, et al. Characterization of Submicron Phase-change Perfluorocarbon Droplets for Extravascular Ultrasound Imaging of Cancer. Ultrasound Med Biol. 2013; 39: 475-489.

9. Gorelikov I, Martin AL, Seo M, et al. Silica-coated quantum dots for optical evaluation of perfluorocarbon droplet interactions with cells. Langmuir. 2011; 27: 15024-15033.

10. Shin SH, Park E-J, Min C, et al. Tracking Perfluorocarbon Nanoemulsion Delivery by ${ }^{19} \mathrm{~F}$ MRI for Precise High Intensity Focused Ultrasound Tumor Ablation. Theranostics. 2017; 7: 562-572.

11. Dewitte H, Geers B, Liang S, et al. Design and evaluation of theranostic perfluorocarbon particles for simultaneous antigen-loading and ${ }^{19} \mathrm{~F}-\mathrm{MRI}$ tracking of dendritic cells. J Control Release. 2013; 169: 141-9.

12. Sheeran PS, Yoo K, Williams R, et al. More Than Bubbles: Creating Phase-Shift Droplets from Commercially Available Ultrasound Contrast Agents. Ultrasound Med Biol. 2016; 43: 531-540.

13. Sheeran PS, Matsuura N, Borden MA, et al. Methods of Generating Sub-Micron Phase-Shift Perfluorocarbon Droplets for Applications in 
Medical Ultrasonography. IEEE Trans Ultrason Ferroelectr Freq Control. 2016; 64: 1-1.

14. Wilson K, Homan K, Emelianov S. Biomedical photoacoustics beyond thermal expansion using triggered nanodroplet vaporization for contrast-enhanced imaging. Nat Commun. 2012; 3: 610-618.

15. Gao Z, Kennedy AM, Christensen DA, et al. Drug-loaded nano/microbubbles for combining ultrasonography and targeted chemotherapy. Ultrasonics. 2008; 48: 260-270.

16. Song KH, Fan AC, Brlansky JT, et al. High efficiency molecular delivery with sequential low-energy sonoporation bursts. Theranostics. 2015; 5: 1419-1427.

17. Matsuura N, Hill ML, Gorelikov I, et al. Development of a targeted CT contrast agent: assessment of cellular interactions using novel integrated optical labels. Proc SPIE. 2009; 7262: 72622A-1-8.

18. Hill ML, Gorelikov I, Niroui F, et al. Towards a nanoscale mammographic contrast agent: development of a modular pre-clinical dual optical/x-ray agent. Phys Med Biol. 2013; 58: 5215-35.

19. Wu SY, Fix SM, Arena CB, et al. Focused ultrasound-facilitated brain drug delivery using optimized nanodroplets: Vaporization efficiency dictates large molecular delivery. Phys Med Biol. 2018; 63:

20. Strohm E, Rui M, Gorelikov I, et al. Vaporization of perfluorocarbon droplets using optical irradiation. Biomed Opt Express. 2011; 2: 1432-42.

21. Goertz DE, Todorova M, Mortazavi O, et al. Antitumor Effects of Combining Docetaxel (Taxotere) with the Antivascular Action of Ultrasound Stimulated Microbubbles. PLoS One. 2012; 7:

22. Ho YJ, Chang YC, Yeh CK. Improving nanoparticle penetration in tumors by vascular disruption with acoustic droplet vaporization. Theranostics. 2016; 6: 392-403.

23. Chang N, Lu S, Qin D, et al. Efficient and controllable thermal ablation induced by short-pulsed HIFU sequence assisted with perfluorohexane nanodroplets. Ultrason Sonochem. 2018; 45: 57-64.

24. Kopechek JA, Park E, Mei C-S, et al. Accumulation of phase-shift nanoemulsions to enhance MR-guided ultrasound-mediated tumor ablation in vivo. J Healthc Eng. 2013; 4: 109-26.

25. Kopechek J a, Park E-J, Zhang Y-Z, et al. Cavitation-enhanced MR-guided focused ultrasound ablation of rabbit tumors in vivo using phase shift nanoemulsions. Phys Med Biol. 2014; 59: 3465-81.

26. Jian J, Liu C, Gong Y, et al. India ink incorporated multifunctional phase-transition nanodroplets for photoacoustic/ultrasound dual-modality imaging and photoacoustic effect based tumor therapy. Theranostics. 2014; 4: 1026-1038.

27. Riess JG. Oxygen Carriers ("Blood Substitutes")Raison d'Etre, Chemistry, and Some Physiology. Chem Rev. 2001; 101: 2797-2920.

28. Castro CI, Briceno JC. Perfluorocarbon-based oxygen carriers: Review of products and trials. Artif Organs. 2010; 34: 622-634.

29. Grimes DR, Warren DR, Warren S. Hypoxia imaging and radiotherapy: bridging the resolution gap. Br J Radiol. 2017; 90: 20160939.

30. Höckel M, Vaupel P. Tumor hypoxia: definitions and current clinical, biologic, and molecular aspects. J Natl Cancer Inst. 2001; 93: 266-276.

31. Wilson WR, Hay MP. Targeting hypoxia in cancer therapy. Nat Rev Cancer. 2011; 11: 393-410.

32. Yu M, Dai M, Liu Q, et al. Oxygen carriers and cancer chemo- and radiotherapy sensitization: Bench to bedside and back. Cancer Treat Rev. 2007: 33: 757-761.

33. Rockwell S, Dobrucki IT, Kim EY, et al. Hypoxia and radiation therapy: past history, ongoing research, and future promise. Curr Mol Med. 2009; 9: 442-58

34. Horsman MR, Mortensen LS, Busk M, et al. Imaging hypoxia to improve radiotherapy outcome. Nat Rev Clin Oncol. 2012; 9: 674-687.

35. Rockwell S. Perfluorochemical emulsions and radiation therapy. Artif Cells Blood Substit Immobil Biotechnol. 1994; 22: 1097-1108.

36. Dowling S, Fischer JJ, Rockwell S. Fluosol and hyperbaric oxygen as an adjunct to radiation therapy in the treatment of malignant gliomas: a pilot study. Biomater Artif Cells Immobilization Biotechnol. 1992; 20: 903-905.

37. Hochberg F, Prados M, Russell C, et al. Treatment of recurrent malignant glioma with BCNU-fluosol and oxygen inhalation. A phase I-II study. J Neurooncol. 1997; 32: 45-55.

38. Evans RG, Kimler BF, Morantz RA, et al. Lack of complications in long-term survivors after treatment with Fluosol and oxygen as an adjuvant to radiation therapy for high-grade brain tumors. Int J Radiat Oncol Biol Phys. 1993; 26: 649-52.

39. Rose C, Lustig R, McIntosh N, et al. A clinical trial of Fluosol DA $20 \%$ in advanced squamous cell carcinoma of the head and neck. Int J Radiat Oncol Biol Phys. 1986; 12: 1325-1327.

40. Vitu-Loas L, Thomas C, Chavaudra N, et al. Radiosensitivity, blood perfusion and tumour oxygenation after perflubron emulsion injection. Radiother Oncol. 1993; 27: 149-155.
41. Teicher BA, Herman TS, Menon K. Enhancement of Fractionated Radiation Therapy by an Experimental Concentrated Perflubron Emulsion ( Oxygent ${ }^{\circledR}$ ) in the Lewis Lung Carcinoma. Biomater Artif Cells Immobil Biotechnol. 1992; 20: 899-902.

42. Long DM, Witt BL, Escobar JA, et al. Effect of emulsion concentration on biodistribution of perflubron in tumor-bearing mice. Biomater Artif Cells Immobilization Biotechnol. 1992; 20: 907-10.

43. Guichard M, Lartigau E, Martin L, et al. Tumor Oxygenation after 1) Carbogen and/or Perflubron Emulsion Administration in Tumor Xenografts 2) Carbogen Administration in Patients. Artif Cells, Blood Substitutes, Biotechnol. 1994; 22: 1355-1360.

44. Rockwell S, Kelley M, Irvin CG, et al. Modulation of tumor oxygenation and radiosensitivity by a perfluorooctylbromide emulsion. Radiother Oncol. 1991; 22: 92-98.

45. Nichols JW, Bae YH. Odyssey of a cancer nanoparticle: from injection site to site of action. Nano Today. 2012; 7: 606-618.

46. Jain RK, Stylianopoulos T. Delivering nanomedicine to solid tumors. Nat Rev Clin Oncol. 2010; 7: 653-664.

47. Fernandes DA, Fernandes DD, Li Y, et al. Synthesis of Stable Multifunctional Perfluorocarbon Nanoemulsions for Cancer Therapy and Imaging. Langmuir. 2016; 32: 10870-10880.

48. Gao M, Liang C, Song X, et al. Erythrocyte-Membrane-Enveloped Perfluorocarbon as Nanoscale Artificial Red Blood Cells to Relieve Tumor Hypoxia and Enhance Cancer Radiotherapy. Adv Mater. 2017; 1701429: $1-7$

49. Xu L, Qiu X, Zhang Y, et al. Liposome encapsulated perfluorohexane enhances radiotherapy in mice without additional oxygen supply. J Transl Med. 2016; 14: 268.

50. Cheng $Y$, Cheng H, Jiang C, et al. Perfluorocarbon nanoparticles enhance reactive oxygen levels and tumour growth inhibition in photodynamic therapy. Nat Commun. 2015; 6: 8785 .

51. Teicher BA, Herman TS, Jones SM. Optimization of perfluorochemical levels with radiation therapy in mice. Cancer Res. 1989; 49: 2693-7.

52. Teicher BA, Rose CM. Oxygen-carrying Perfluorochemical Emulsion as an Adjuvant to Radiation Therapy in Mice. Cancer Res. 1984; 44: 4285-4288.

53. Zheng J, Klinz SG, De Souza R, et al. Longitudinal tumor hypoxia imaging with [(18)F]FAZA-PET provides early prediction of nanoliposomal irinotecan (nal-IRI) treatment activity. EJNMMI Res. 2015; 5: 57.

54. Song CW, Lee I, Hasegawa $\mathrm{T}$, et al. Increase in $\mathrm{pO} 2$ and radiosensitivity of tumors by Fluosol-DA (20\%) and carbogen. Cancer Res. 1987; 47: $442-446$.

55. Martin AL, Seo M, Williams R, et al. Intracellular Growth of Nanoscale Perfluorocarbon Droplets for Enhanced Ultrasound-Induced Phase-Change Conversion. Ultrasound Med Biol. 2012; 38: 1799-1810.

56. Czarnota GJ, Karshafian R, Burns PN, et al. Tumor radiation response enhancement by acoustical stimulation of the vasculature. 2012; .

57. Matsuura N, Koonar E, Zhu S, et al. Inducing antivascular effects in tumors with ultrasound stimulated micron-sized bubbles. 2015; 3-6

58. Higgins LH, Withers HG, Garbens A, et al. Hypoxia and the metabolic phenotype of prostate cancer cells. Biochim Biophys Acta - Bioenerg. 2009; 1787: 1433-1443.

59. Tran LBA, Bol A, Labar D, et al. Predictive value of $18 \mathrm{~F}-\mathrm{FAZA}$ PET imaging for guiding the association of radiotherapy with nimorazole: $\mathrm{A}$ preclinical study. Radiother Oncol. 2015; 114: 189-194.

60. Li X, Qin F, Yang $\mathrm{L}$, et al. Sulfatide-containing lipid perfluorooctylbromide nanoparticles as paclitaxel vehicles targeting breast carcinoma. Int J Nanomedicine. 2014; 9: 3971-3985.

61. Faustino-Rocha A, Oliveira PA, Pinho-Oliveira J, et al. Estimation of rat mammary tumor volume using caliper and ultrasonography measurements. Lab Anim (NY). 2013; 42: 217-224.

62. Maldiney T, Richard C, Seguin J, et al. Effect of core diameter, surface coating, and PEG chain length on the biodistribution of persistent luminescence nanoparticles in mice. ACS Nano. 2011; 5: 854-862.

63. Meng F, Wang J, Ping Q, et al. Quantitative Assessment of Nanoparticle Biodistribution by Fluorescence Imaging, Revisited. ACS Nano. 2018; 12: 6458-6468.

64. Kumar V, Mundra V, Peng Y, et al. Pharmacokinetics and biodistribution of polymeric micelles containing miRNA and small-molecule drug in orthotopic pancreatic tumor-bearing mice. Theranostics. 2018; 8: 4033-4049.

65. Lee MJ-E, Veiseh O, Bhattarai N, et al. Rapid Pharmacokinetic and Biodistribution Studies Using Cholorotoxin-Conjugated Iron Oxide Nanoparticles: A Novel Non-Radioactive Method. PLoS One. 2010; 5: e9536.

66. Sorger D, Patt M, Kumar P, et al. [18 F]Fluoroazomycinarabinofuranoside (18 FAZA) and [18 F]Fluoromisonidazole (18 FMISO): A comparative study of their 
selective uptake in hypoxic cells and PET imaging in experimental rat tumors. Nucl Med Biol. 2003; 30: 317-326.

67. Metran-Nascente C, Yeung I, Vines DC, et al. Measurement of Tumor Hypoxia in Patients with Advanced Pancreatic Cancer Based on 18F-Fluoroazomyin Arabinoside Uptake. J Nucl Med. 2016; 57: 361-366.

68. Busk M, Munk OL, Jakobsen S, et al. Assessing hypoxia in animal tumor models based on pharmocokinetic analysis of dynamic FAZA PET. Acta Oncol. 2010; 49: 922-933.

69. Liang $X$, Fang L, Li X, et al. Activatable near infrared dye conjugated hyaluronic acid based nanoparticles as a targeted theranostic agent for enhanced fluorescence/CT/photoacoustic imaging guided photothermal therapy. Biomaterials. 2017; 132: 72-84.

70. Vu-Quang H, Vinding MS, Nielsen T, et al. Theranostic tumor targeted nanoparticles combining drug delivery with dual near infrared and $19 \mathrm{~F}$ magnetic resonance imaging modalities. Nanomedicine Nanotechnology, Biol Med. 2016; 12: 1873-1884.

71. Ke H, Wang J, Tong S, et al. Gold nanoshelled liquid perfluorocarbon magnetic nanocapsules: A nanotheranostic platform for bimodal ultrasound/magnetic resonance imaging guided photothermal tumor ablation. Theranostics. 2014; 4: 12-23.

72. Diou O, Tsapis N, Giraudeau C, et al. Long-circulating perfluorooctyl bromide nanocapsules for tumor imaging by 19FMRI. Biomaterials. 2012; 33: 5593-5602.

73. Fraker CA, Mendez AJ, Inverardi L, et al. Optimization of perfluoro nano-scale emulsions: The importance of particle size for enhanced oxygen transfer in biomedical applications. Colloids Surfaces B Biointerfaces. 2012; 98: 26-35.

74. Liu L, Bagia C, Janjic JM. The First Scale-Up Production of Theranostic Nanoemulsions. Biores Open Access. 2015; 4: 218-28.

75. Cornelus C, Giulieri F, Riess MPK au JG. Impact of the structure of phospholipid dispersions on the stability of fluorocarbon/phospholipid emulsions for biomedical uses. Colloids Surfaces A Physicochem Eng Asp. 1993; 70: 233-238.

76. Postel M, Riess JG, Weers JG. Fluorocarbon emulsions--the stability issue. Artif Cells Blood Substit Immobil Biotechnol. 1994; 22: 991-1005.

77. Riess JG, Weers JG. Emulsions for biomedical uses. Curr Opin Colloid Interface Sci. 1996; 1: 652-659.

78. Brannon-Peppas L, Blanchette JO. Nanoparticle and targeted systems for cancer therapy. Adv Drug Deliv Rev. 2012; 64: 206-212.

79. Neubauer AM, Sim H, Winter PM, et al. Nanoparticle pharmacokinetic profiling in vivo using magnetic resonance imaging. Magn Reson Med. 2008; 60: 1353-1361.

80. Lee YH, Yeh YL. Reduction of oxygen inhibition effect for microalgal growth using fluoroalkylated methoxy polyethylene glycol-stabilized perfluorocarbon nano-oxygen carriers. Process Biochem. 2015; 50: 1119-1127.

81. Khattak SF, Chin K, Bhatia SR, et al. Enhancing oxygen tension and cellular function in alginate cell encapsulation devices through the use of perfluorocarbons. Biotechnol Bioeng. 2007; 96: 156-166.

82. White JC, Godsey ME, Bhatia SR. Perfluorocarbons enhance oxygen transport in alginate-based hydrogels. Polym Adv Technol. 2014; 25: 1242-1246.

83. Daşu A, Toma-Daşu I, Karlsson M. Theoretical simulation of tumour oxygenation and results from acute and chronic hypoxia. Phys Med Biol. 2003; 48: 2829-42

84. Grimes DR, Fletcher AG, Partridge M. Oxygen consumption dynamics in steady-state tumour models. R Soc open Sci. 2014; 1: 140080.

85. Grimes DR, Kannan P, Warren DR, et al. Estimating oxygen distribution from vasculature in three-dimensional tumour tissue. J R Soc Interface. 2016; 13: 638-648.

86. Zoabi N, Golani-Armon A, Zinger A, et al. The evolution of tumor-targeted drug delivery: From the EPR effect to nanoswimmers. Isr J Chem. 2013; 53: 719-727.

87. Kergoat H, Faucher C. Effects of oxygen and carbogen breathing on choroidal hemodynamics in humans. Investig Ophthalmol Vis Sci. 1999; 40: 2906-2911.

88. Dunn TJ, Braun RD, Rhemus WE, et al. The effects of hyperoxic and hypercarbic gases on tumour blood flow. Br J Cancer. 1999; 80: 117-26.

89. Radisic $\mathrm{M}$, Deen $\mathrm{W}$, Langer $\mathrm{R}$, et al. Mathematical model of oxygen distribution in engineered cardiac tissue with parallel channel array perfused with culture medium containing oxygen carriers. Am J Physiol Heart Circ Physiol. 2005; 288: H1278-1289.

90. Mates vanLöbensels E, Anderson JC, Hildebrandt J, et al. Modeling diffusion limitation of gas exchange in lungs containing perfluorocarbon. J Appl Physiol. 1999; 86: 273-84.

91. Jiang $\mathrm{W}$, Delahunty IM, Xie J. Oxygenating the way for enhanced chemophototherapy. Theranostics. 2018; 8: 3870-3871.

92. Li X, Kwon N, Guo T, et al. Innovative Strategies for Hypoxic-Tumor Photodynamic Therapy. Angew Chemie - Int Ed. 2018; 11522-11531.
93. Day RA, Estabrook DA, Logan JK, et al. Fluorous photosensitizers enhance photodynamic therapy with perfluorocarbon nanoemulsions. Chem Commun. 2017; 53: 13043-13046.

94. Wang J, Liu L, You Q, et al. All-in-one theranostic nanoplatform based on hollow MoSx for photothermally-maneuvered oxygen self-enriched photodynamic therapy. Theranostics. 2018; 8: 955-971.

95. Horsman MR, Lindegaard JC, Grau C, et al. Dose-response modifiers in radiation therapy. Clin Radiat Oncol. 2011;

96. Price CD, El-Badri NS, Haas DK a., et al. The Evaluation of the Efficacy of Oxygent ${ }^{\circledR}$ as an Oxygen-Carrying Substitute on Cerebral Blood Flow. Open Hematol J. 2008; 2: 62-66.

97. Robinson S, Rodrigues L, Stubbs M, et al. Effects of different levels of hypercapnic hyperoxia on tumour GRE image intensity and arterial blood gases. Proc ISMRM 7th Ann Meet. 1999; 19: 1372. 